\title{
Coefficient Distribution Method for Minimum Weight Design of Large-Span Statically Determinate Trusses under the Constraint of Allowable Displacement
}

\author{
Wenfeng Du*, Qi Liu, Kang Yuan and Jiyuan Ma \\ Institute of Steel and Spatial Structure in School Civil Engineering and Architecture, Henan University, Kaifeng 475004 China \\ ${ }^{*}$ Corresponding author
}

\begin{abstract}
To achieve the minimum weight design of large-span statically determinate trusses under displacement constraint, a method called the coefficient distribution method was proposed. The calculation formula of the displacement for statically determinate trusses was provided at first. Then the critical condition of minimum weight design satisfying the allowable displacement was deduced using Cauchy Inequality. At last, the distribution coefficient of material consumption, which was used to determine the proportional relationship of weight of each member in a truss, was defined. An example was analyzed using the coefficient distribution method. Study results show that the coefficient distribution method is correct, reliable and effective.
\end{abstract}

Keywords - the allowable displacement; the minimum weight design; the coefficient distribution method; large-span determinate truss

\section{INTRODUCTION}

The truss has been one of the most common used structures since ancient times in structural engineering history [1]. Using such a design is advantageous in that it can form a stable large system by assembling small members connected at their ends by hinges [2]. Under the action of joints loads members in a truss are subjected only to axial forces, so it is simple to design and construct trusses [3]. However, how to minimize the weight during designing a truss has been a resurgence of interest for past 30 years $[4,5]$.

The design optimization of trusses can be divided three levels. The first is the level of cross section. The weight of a truss member depends directly on its cross section. Optimization using sizing variables such as areas or thickness has been the most popular form [6]. The second is the level of members. Because of the problem of instability, choose of section shapes and length-width-ratio of compression members become complex [7]. The third is the level of the structure. The structural configuration and the layout of each member have important influences to the weight of a truss. Topology optimization has been studied and researched worldwide $[8,9]$.

Depending on the number of variables, such an iterative technique may require a large number of structural analyses that are generally costly and time consuming. Therefore, if the condition of the critical value corresponding to the minimize weight with displacement constraints can be found through the theoretical analysis, it is meaningful to guide the design. A method called the coefficient distribution method was proposed by theoretical analysis in this paper to reach the minimum weight design of large-span statically determinate trusses under the displacement constraint.

\section{CoEFFicient Distribution Method}

\section{A. Fundamental Assumption}

(1)The joints of the large-span truss are regarded as ideal hinges. Most of the trusses in practical engineering conform to this assumption.

(2) The trusses are made of the linear elastic material. The common used material, such as steel, reinforced concrete, aluminum, and wood follow this assumption.

(3)Static loads are only applied on joints. Therefore, there are only axial forces in members. Most trusses usually can use this assumption.

\section{B. Calculation Formula of Displacement}

The calculation formula of displacement of the truss structure is:

$$
\Delta=\sum_{i=1}^{n} \frac{N_{i} \overline{N_{i}} L_{i}}{E_{i} A_{i}}
$$

Where, $\Delta$ is the displacement of each joint, $i$ is the number of each member, $N_{i}$ is the axial force of member $i$ under static loads, $\overline{N_{i}}$ is the axial force of member $i$ under the virtual unit load, $L_{i}$ is the length of the member $i, A_{1}$ is the cross section area of the member $i, E_{i}$ is the modulus of elasticity.

\section{Mathematical Optimization Model of Minimum Weight}

The weight of each member can be calculated by the cross section area and the length. Therefore, the weight of a truss is: 


$$
W=\rho g \sum_{i=1}^{n} L_{i} A_{i}
$$

Where, $g$ is the acceleration of gravity, and $\rho$ is the density of material. Due to the acceleration of gravity and the density of material are constant, $\rho g$ can be regarded as a constant $C$ for simple, $C=1$.

Then, the mathematical optimization model of minimum weight that need satisfy the requirement of allowable displacement is:

$$
\left.\begin{array}{l}
\text { Min, } W \\
\text { s.t. } \Delta=\sum_{i=1}^{n} \frac{N_{i} \overline{N_{i}} L_{i}}{E A_{i}} \leq[\Delta] \\
\operatorname{var} 1 \leq i \leq n, i \in N
\end{array}\right\}
$$

deflection.

\section{Solution of Optimization Design Model}

When the structural weight is lightest, the displacement of structure equals the allowable displacement theoretically, namely $\Delta=[\Delta]$.

After that, to find the minimum value of $W$, we can calculate the minimum value of $\mathrm{W} \cdot \Delta$ equivalently.

$$
W \Delta=\left(L_{1} A_{1}+L_{2} A_{2}+\cdot \cdot+L_{1} A_{1}+\cdot \cdot+L_{n} A_{n}\right)\left(\frac{N \bar{N} L_{1}^{2}}{E A_{1}}+\frac{N_{2} \overline{N_{2}} L_{2}^{2}}{E A_{2} L_{2}}+\cdot \cdot+\frac{N_{i} N_{i} L_{1}^{2}}{E A_{1} L_{1}}+\cdot \cdot+\frac{N_{n} \bar{N} L_{n}^{2}}{E A_{n} L_{n}}\right)
$$

$$
\text { According to the Cauchy Inequality. }
$$

Where, $W$ is the objective function, $[\Delta]$ is allowable

$$
\begin{aligned}
& W \Delta \geq\left(\sqrt{L_{1} A_{1}} * \sqrt{\frac{N_{1} \overline{N_{1}} L_{1}^{2}}{E A_{1} L_{1}}}+\sqrt{L_{2} A_{2}} * \sqrt{\frac{N_{2} \overline{N_{2}} L_{2}{ }^{2}}{E A_{2} L_{2}}}+\cdots+\sqrt{L_{i} A_{1}} * \sqrt{\frac{N_{i} \overline{N_{i}} L_{i}^{2}}{E A_{i} L_{i}}}+\cdots+\sqrt{L_{n} A_{n}} * \sqrt{\frac{N_{n} \overline{N_{n}} L_{n}{ }^{2}}{E A_{n} L_{n}}}\right)^{2} \\
& =\left(\sqrt{\frac{N_{1} \overline{N_{1}} L_{1}^{2}}{E}}+\sqrt{\frac{N_{2} \overline{N_{2}} L_{2}^{2}}{E}}+\cdots+\sqrt{\frac{N_{i} \overline{N_{i}} L_{i}{ }^{2}}{E}}+\cdots+\sqrt{\frac{N_{n} \overline{N_{n}} L_{n}{ }^{2}}{E}}\right)^{2}
\end{aligned}
$$

The critical condition for equality is as following.

$$
\sqrt{\frac{N_{i} \overline{N_{i}} L_{i}^{2}}{E A_{i} L_{i}}} / \sqrt{L_{i} A_{i}}=\sqrt{\frac{N_{j} \overline{N_{j}} L_{j}^{2}}{E A_{j} L_{j}}} / \sqrt{L_{j} A_{j}},(i=j)
$$

Then,

$$
L_{i} A_{i} / L_{j} A_{j}=\sqrt{N_{i} \overline{N_{i}} L_{i}^{2}} / \sqrt{N_{j} \overline{N_{j}} L_{j}^{2}}=W_{i} / W_{j}
$$

The formula (7) indicates that there exists a certain proportion among individual member weight for large-span determinate trusses to achieve the minimum weight design meeting the requirement of the allowable displacement. So, we can define a coefficient $\mu$ which is the ratio between the weight of each member and the structural total weight.

$$
\mu_{i}=\sqrt{N_{i} \overline{N_{i}} L_{i}^{2}} / \sum_{i=1}^{n} \sqrt{N_{i} \overline{N_{i}} L_{i}^{2}}
$$

Where, $\mu$ is the ratio between the weight of member $i$ and the total weight. It denotes that when the total material usage are determined, if the amount of material of each member in a statically determinate truss is decided by the distribution coefficient, the maximum structural stiffness is obtained. In another word, under the constraint of allowable displacement, if the proportion of material usage of each member in a statically determinate truss is decided by this distribution coefficient, the material consumption of structure is lightest. Therefore, $\mu$ can be defined as the distribution coefficient.

Substitute formula (8) into formula (1) and formula (2), then we can obtain the minimum weight of a statically determinate truss:

$$
W=\left(\sum \frac{N_{i} \bar{N}_{i} L_{i}^{2}}{E u_{i}}\right) /[\Delta]
$$

\section{E. Calculation Process of Coefficient Distribution Method}

According to the previous derivation, when the coefficient distribution method was used to design large-span statically determinate trusses, following steps can be used.

(1) Applying a unit load at the joint where the maximum displacement may happen;

(2) Calculating the axial force of each member $i$ under the unit load; 
(3) Calculating the axial force of each member $i$ under external loads;

(4) Calculating the distribution coefficient $\mu$ according to formula (8);

(5) Calculating the minimum consumption of material using formula (9);

(6) Finally, the section area of each member $i$ is calculated.

\section{AN EXAMPLE OF APPLICATION}

\section{A. Project Introduction}

The structural model of a statically determinate truss is shown in Fig.1. The span and height of the structural model is $24 \mathrm{~m}$ and $4 \mathrm{~m}$ respectively. There are 45 members in the structure and the number of each element is shown in Fig.1. The total number of joints is 24 , and two ends of the structure are simply supported. The steel Q235B is used, whose elastic modulus, yield strength and density are $206 \mathrm{KN} / \mathrm{mm} 2,215 \mathrm{MPa}$, and $7850 \mathrm{~kg} / \mathrm{m}^{3}$ respectively. The vertical load of $412 \mathrm{kN}$ is added on each joint. The allowable displacement $[\Delta]$ equals to $60 \mathrm{~mm}(\mathrm{~L} / 400, \mathrm{~L}$ is the structural span).

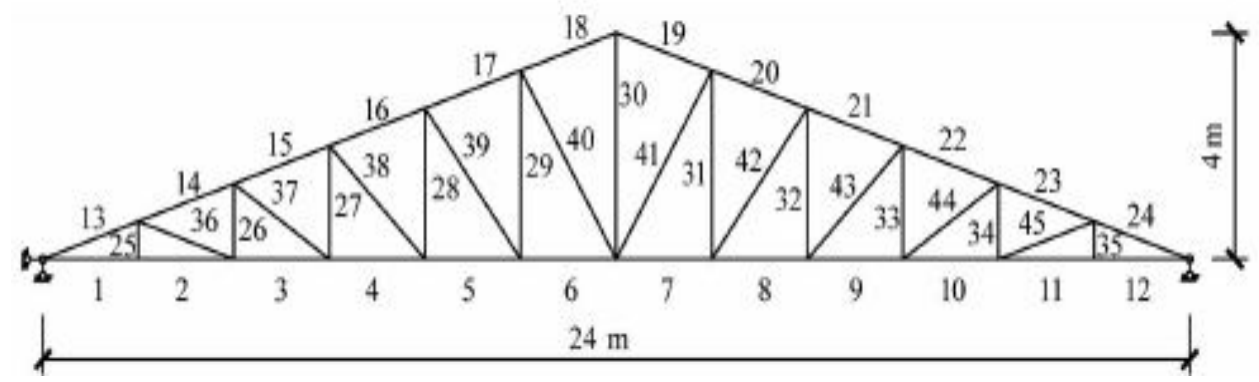

FIGURE I. STRUCTURAL MODEL AND THE NUMBER OF EACH MEMBER

\section{B. Calculation and Comparison}

At first, the structure was designed using the full stress design method and the steel consumption of each member was shown in the third column in Table 1. And according to the equation (1), the structural displacement was calculated to be $83.15 \mathrm{~mm}$ which exceeds the allowable displacement $(60 \mathrm{~mm})$. Then, to satisfy the requirement of the allowable displacement, the section area of each member was expanded by the proportion $83.15 / 60$, and the structural displacement was recalculated, until it met the requirement of allowable displacement. Both the amount of steel used for each member of the structure and the total amount of the steel are shown in the fourth column of Table 1 .

After that, the structure was designed according to the coefficient distribution method proposed in this paper. First, the axial force of every element under the same load was calculated, as shown in the fifth column in Table 1. And a unit force was applied on the lower chord joint in the middle of the truss, as shown in the sixth column in Table 1 . Then, formula (9) was applied to calculate the total amount of steel and the distribution coefficient of each member can be got form formula (8). The total amount of steel is assigned to each member according to its distribution coefficient, as shown in the 7th column in Table 1.

Then the displacement of the structure can be calculated according to the formula (1), which is equal to the allowed displacement $[\Delta]$.

The data in the 8th column is the amount of steel that meets the requirement of strength on the basis of the 7 th column 
TABLE I. THE CALCULATED RESULTS

\begin{tabular}{|c|c|c|c|c|c|c|c|}
\hline $\begin{array}{l}\text { element } \\
\text { number } \\
\text { (i) }\end{array}$ & $\begin{array}{l}\text { length of } \\
\text { each } \\
\text { member }(\mathrm{Li} \\
/ \mathrm{m})\end{array}$ & $\begin{array}{l}\text { steel consumption } \\
\text { using the Full } \\
\text { Stress Design } \\
\text { Method }(\mathrm{W} / \mathrm{kg})\end{array}$ & $\begin{array}{l}\text { steel consumption } \\
\text { after adjustment } \\
(\mathrm{W} / \mathrm{kg})\end{array}$ & $\begin{array}{lr}\text { Axial } & \text { forces } \\
\text { under } & \text { loads } \\
(\mathrm{Ni} / \mathrm{kN}) & \end{array}$ & $\begin{array}{l}\text { Axial forces } \\
\text { under the unit } \\
\text { load. } \\
(\overline{\mathrm{N}} \mathrm{i} / \mathrm{kN})\end{array}$ & $\begin{array}{l}\text { steel consumption } \\
\text { using the Coefficient } \\
\text { Method }(\mathrm{W} / \mathrm{kg})\end{array}$ & $\begin{array}{l}\text { steel consumption } \\
\text { after adjustment } \\
(\mathrm{W} / \mathrm{kg})\end{array}$ \\
\hline 1 & 2 & 5.290535 & 7.365394 & 72.45 & 1.5 & 6.663822 & 6.663822 \\
\hline 2 & 2 & 5.290535 & 7.365394 & 72.45 & 1.5 & 6.663822 & 6.663822 \\
\hline 3 & 2 & 4.830488 & 6.724925 & 66.15 & 1.5 & 6.367502 & 6.367502 \\
\hline 4 & 2 & 4.370442 & 6.084456 & 59.85 & 1.5 & 6.056703 & 6.056703 \\
\hline 5 & 2 & 3.910395 & 5.443987 & 53.55 & 1.5 & 5.729067 & 5.729067 \\
\hline 6 & 2 & 3.450349 & 4.803518 & 47.25 & 1.5 & 5.381522 & 5.381522 \\
\hline 7 & 2 & 3.542358 & 4.931612 & 48.51 & 1.5 & 5.452803 & 5.452803 \\
\hline 8 & 2 & 4.140419 & 5.764222 & 56.7 & 1.5 & 5.895162 & 5.895162 \\
\hline 9 & 2 & 4.830488 & 6.724925 & 66.15 & 1.5 & 6.367502 & 6.367502 \\
\hline 10 & 2 & 5.290535 & 7.365394 & 72.45 & 1.5 & 6.663822 & 6.663822 \\
\hline 11 & 2 & 5.750581 & 8.005864 & 78.75 & 1.5 & 6.947515 & 6.947515 \\
\hline 12 & 2 & 5.750581 & 8.005864 & 78.75 & 1.5 & 6.947515 & 6.947515 \\
\hline 13 & 2.11 & 5.883433 & 8.190817 & \begin{tabular}{|l|}
-76.369 \\
\end{tabular} & 1.581139 & 7.410621 & 7.410621 \\
\hline 14 & 2.11 & 5.37183 & 7.478572 & -69.7282 & 1.581139 & 7.081093 & 7.081093 \\
\hline 15 & 2.11 & 4.860227 & 6.766327 & -63.0874 & 1.581139 & 6.735463 & 6.735463 \\
\hline 16 & 2.11 & 4.348624 & 6.054082 & -56.4467 & 1.581139 & 6.37111 & 6.37111 \\
\hline 17 & 2.11 & 3.837021 & 5.341837 & -49.8059 & 1.581139 & 5.984616 & 5.984616 \\
\hline 18 & 2.11 & 3.325418 & 4.629592 & -43.1651 & 1.581139 & 5.571374 & 5.571374 \\
\hline 19 & 2.11 & 3.325418 & 4.629592 & -43.1651 & 1.581139 & 5.571374 & 5.571374 \\
\hline 20 & 2.11 & 3.939342 & 5.484286 & -51.134 & 1.581139 & 6.063886 & 6.063886 \\
\hline 21 & 2.11 & 4.604426 & 6.410205 & -59.767 & 1.581139 & 6.555818 & 6.555818 \\
\hline 22 & 2.11 & 5.37183 & 7.478572 & -69.7282 & 1.581139 & 7.081093 & 7.081093 \\
\hline 23 & 2.11 & 5.883433 & 8.190817 & $\begin{array}{l}-76.369 \\
\end{array}$ & 1.581139 & 7.410621 & 7.410621 \\
\hline 24 & 2.11 & 6.395035 & 8.903062 & -83.0098 & 1.581139 & 7.726106 & 7.726106 \\
\hline 25 & 0.67 & 0 & 0 & 0 & 0 & 0 & 0 \\
\hline 26 & 1.33 & 0.101977 & 0.141971 & 2.1 & 0 & 0 & 0.101977 \\
\hline 27 & 2 & 0.306698 & 0.426979 & 4.2 & 0 & 0 & 0.306698 \\
\hline 28 & 2.67 & 0.614162 & 0.855026 & 6.3 & 0 & 0 & 0.614162 \\
\hline 29 & 3.33 & 1.021303 & 1.421841 & 8.4 & 0 & 0 & 1.021303 \\
\hline 30 & 4 & 3.373674 & 4.696773 & 23.1 & 1 & 6.144613 & 6.144613 \\
\hline 31 & 3.33 & 1.327694 & 1.848394 & 10.92 & 0 & 0 & 1.327694 \\
\hline 32 & 2.67 & 0.921243 & 1.282539 & 9.45 & 0 & 0 & 0.921243 \\
\hline 33 & 2 & 0.306698 & 0.426979 & 4.2 & 0 & 0 & 0.306698 \\
\hline 34 & 1.33 & 0.101977 & 0.141971 & 2.1 & 0 & 0 & 0.101977 \\
\hline 35 & 0.67 & 0 & 0 & 0 & 0 & 0 & 0 \\
\hline 36 & 2.11 & 0.511603 & 0.712245 & -6.64078 & 0 & 0 & 0.511603 \\
\hline 37 & 2.4 & 0.663489 & 0.923698 & -7.57166 & 0 & 0 & 0.663489 \\
\hline 38 & 2.83 & 0.920605 & 1.28165 & -8.90955 & 0 & 0 & 0.920605 \\
\hline 39 & 3.33 & 1.276629 & 1.777302 & -10.5 & 0 & 0 & 1.276629 \\
\hline 40 & 3.89 & 1.73916 & 2.42123 & -12.245 & 0 & 0 & 1.73916 \\
\hline 41 & 3.89 & 2.086992 & 2.905476 & -14.694 & 0 & 0 & 2.086992 \\
\hline 42 & 3.33 & 1.659618 & 2.310492 & -13.65 & 0 & 0 & 1.659618 \\
\hline 43 & 2.83 & 1.380907 & 1.922476 & -13.3643 & 0 & 0 & 1.380907 \\
\hline 44 & 2.4 & 0.663489 & 0.923698 & -7.57166 & 0 & 0 & 0.663489 \\
\hline 45 & 2.11 & $\begin{array}{l}0.511603 \\
\end{array}$ & 0.712245 & -6.64078 & 0 & 0 & 0.511603 \\
\hline sum & $\overline{-}$ & 133.0833 & 185.2763 & - & $\overline{-}$ & 160.8445 & $\begin{array}{l}176.9604 \\
\end{array}$ \\
\hline
\end{tabular}

\section{Analysis and Discussion}

According to the results of this project, it can be known that when the strength requirement is met only, the minimum amount of steel calculated by full stress design method is $133.108 \mathrm{~kg}$. When stiffness requirement is met only, the minimum amount of steel calculated by the coefficient distribution method is $160.184 \mathrm{~kg}$.

When both the strength and stiffness requirements are met, the minimum amount of steel is $176.196 \mathrm{~kg}$, which is obtained using the coefficient distribution method. It is to meet the stiffness requirement at first and then to adjust the members who cannot meet the strength requirement to the values calculated using the full stress design method. If the process above is reversed, the outcome will be $185.128 \mathrm{~kg}$. Table. 1 shows that under the constrains of strength and stiffness, the results according to the coefficient distribution method can save the amount of steel by $4.5 \%$ compared with the results of the full stress design method. Fig. 2 shows the situation of the distribution coefficient of each member. 


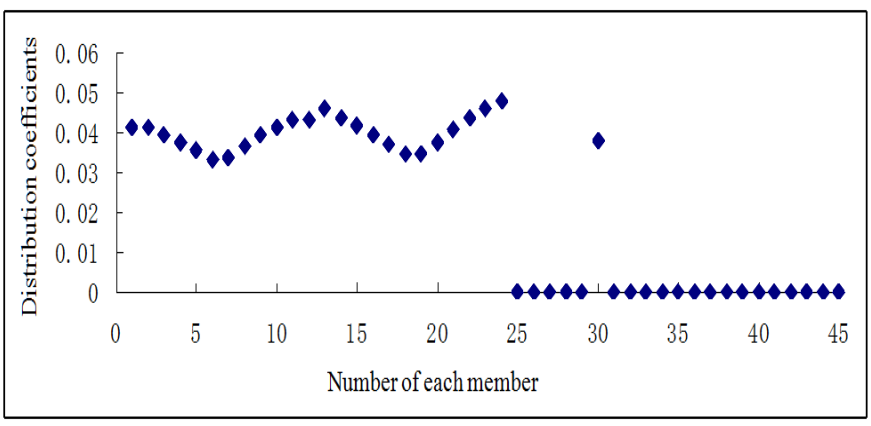

FIGURE II. DISTRIBUTION COEFFICIENT OF EACH MEMBER

The distribution coefficients of these members numbering from 1 to 24 and 30 are not zero (about 0.04), and the distribution coefficients of the rest members are all zero. That indicates these members did not affect the vertical displacement of the middle joint, because the internal forces of these members under the unit load are zero. These members whose distribution coefficients are not equal to zero are located at the top chord, the lower chord and the middle vertical web. The distribution coefficient demonstrates that the influence of each member to structural displacement, and the member numbering 24 and 13 have the largest influence on vertical displacement because their distribution coefficients are both the largest(about 0.048). They are located at the top chord connecting with supports. While, the other webs in the truss have no effect on the structural displacements because their distribution coefficients are zero.

Because there are many kinds of trusses applied in practical engineering, three common forms in Fig. 3 were chosen to make a further analysis. Their spans were $4 \mathrm{~m}, 8 \mathrm{~m}, 12 \mathrm{~m}, 16 \mathrm{~m}, 20 \mathrm{~m}$, $24 \mathrm{~m}, 28 \mathrm{~m}, 32 \mathrm{~m}, 36 \mathrm{~m}$ separately, and the length of each lower chord member is $2 \mathrm{~m}$.

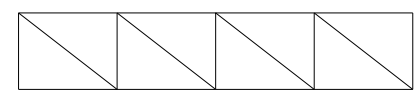

(a)

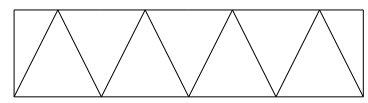

(b)

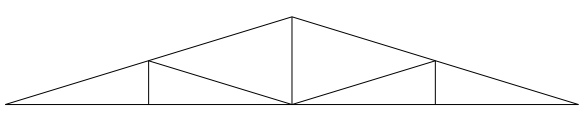

(c)

FIGURE III. THREE KINDS OF TYPICAL TRUSS STRUCTURE

Using the same process with the example in Fig.1, these three typical kinds of trusses were calculated respectively. The results show that these three trusses designed with the coefficient distribution method can save the amount of steel compared with the full stress design method, as shown in Fig.4.
It is obvious the effect of the coefficient distribution method depend on the structural spans. The larger the span is, the more amount of steel saved in the three kinds of trusses are from Fig.4.

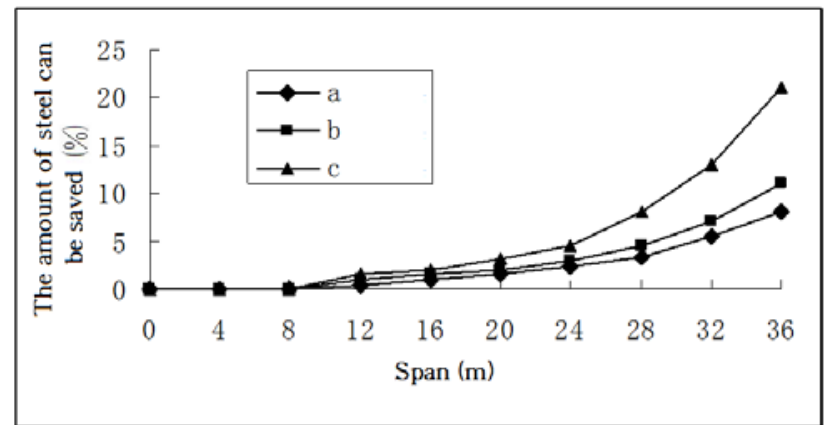

FIGURE IV. THE SAVED STEEL WITH THE STRUCTURAL SPAN OF THE THREE TYPICAL TRUSSES

Type $\mathrm{C}$ truss can save the most amount of steel obviously, when the span is $36 \mathrm{~m}$, the saved steel consumption can reach to $21 \%$. It indicates the coefficient distribution method is very suitable for applying on triangle shape trusses obviously. When the span is small, the results of the full stress design method and those of the full displacement coefficient distribution method are the same, because the structural stiffness does not control the design of the structure.

\section{CONCLUSION}

(1)This article proposed the coefficient distribution method to realize the minimum weight design of large-span statically determinate trusses under the constraint of allowable displacement. This is a design method of structural optimization to improve the structural stiffness meeting the requirement of displacements. The coefficient distribution method has theoretical and practical applications on the large span light steel trusses whose designs are decided by stiffness.

(2)The coefficient distribution method is based on the Cauchy Inequality. The critical condition shows that there exists a certain proportion relationship among individual member weight for statically determinate trusses to achieve the minimum weight design meeting the requirement of the allowable displacement.

(3)Results of comparison between the coefficient distribution method and the full stress method, the former is more economic than the latter for trusses. To the truss with triangle shape, $21 \%$ steel can be saved using the coefficient distribution method when the structural span is $36 \mathrm{~m}$.

\section{REFERENCES}

[1] L. Gil, A. Andreu, Shape and cross-section optimization of a truss structure, J. Computers \& Structures. 79(2001) 681-689.

[2] L. Lamberti, An efficient simulated annealing algorithm for design optimization of truss structures, J. Computers \& Structures, 86(2008)1936-1953.

[3] V. Plevris, M. Papadrakakis, A hybrid particle swarm-gradient algorithm for global structural optimization, J. Computer-Aided Civil and Infrastructure Engineering, 26(2011)48-68. 
[4] K. Mela, Resolving issues with member buckling in truss topology optimization using a mixed variable approach, J. Structural and Multidisciplinary Optimization, 50(2014) 1037-1048.

[5] A. Cerveira, A. Agra, F. Bastos, J. Gromicho, A new Branch and Bound method for a discrete truss topology design problem, J. Computational Optimization and Applications, 54(2013)163-168.

[6] M. Jalalpour, T. Igusa, J.K. Guest, Optimal design of trusses with geometric imperfections: Accounting for global instability, J. International Journal of Solids and Structures, 48(2008)3011-3022.

[7] R.J. Balling, R.R. Briggs, K. Gillman. Multiple optimum size/shape/topology designs for skeletal structures using a genetic algorithm, J. Journal of Structural engineering, 132(2006)1158-1165.

[8] D. Wang, W.H Zhang, J.S Jiang, Truss shape optimization with multiple displacement constraints, J. Computer Methods in Applied Mechanics and Engineering, 191(2002)3597-3612.

[9] A.R. Yildiz, A novel hybrid immune algorithm for global optimization in design and manufacturing, J. Robotics and Computer-Integrated Manufacturing, 25(2008)227-261. 